Research Article

\title{
Simulation Study of Li Doped Carbon Nanotube as a Carrier System for Aspirin in Aqueous Media
}

\author{
Yasaman Ketabi ${ }^{1}$, Sepideh Ketabi ${ }^{2}$ \\ ${ }^{1}$ Shahid Beheshti University of Medical Science, Tehran, Iran \\ ${ }^{2}$ Department of Chemistry, East Tehran Branch, Islamic Azad University, Tehran, Iran \\ Corresponding author: E-mail: sepidehketabi@yahoo.com
}

Received: Feb. 9, 2015; Accepted: Apr. 10, 2015; Published: Apr. 17, 2015.

Citation: Yasaman Ketabi and Sepideh Ketabi. Simulation Study of Li Doped Carbon Nanotube as a Carrier System for Aspirin in Aqueous Media. Nano Biomed. Eng. 20I5, 7(I), 20-27.

DOI: $10.5101 / n$ be.v7il.p20-27.

\begin{abstract}
Potential application of CNT in medical science is the essence of this research. Interaction of CNT and Li doped CNT with Aspirin as a drug was studied in gas phase and in aqueous solution. Aspirin anion and neutral Aspirin were considered in physiological media. Each species was first modeled by quantum mechanical calculations, and then their properties in aqueous solution were studied by applying Monte Carlo simulations. The results of density functional calculations in gas phase show that only Li doped CNT can interact with Aspirin. Computation of solvation free energies in water showed that solubility of the complexes of Aspirin species with Li doped CNT was larger than CNT. Enhancement of the solubility of CNT improves the medicinal applications of these materials. Calculation of complexation free energies indicated that only Li doped CNT produce the stable complex in aqueous solution. So CNTLi can be appropriate system as a possible effective drug transporter for Aspirin delivery.
\end{abstract}

Keywords: Aspirin; CNT; CNTLi; Solvation free energy; MC simulation; DFT

\section{Introduction}

The well-defined shape and size of CNTs make them attractive candidates for applications in the biotechnological sciences [1-4] many theoretical and experimental studies have been done on various nanoscopic phenomena such as protection and confinement of molecular species as well as transport of molecules [5-7]. CNTs have recently become promising materials in various biological applications such as drug delivery [8-11], tumor therapy [12], biosensors [13], and templates for biomolecule assembly [14].
The two limitations of CNTs usage in biological systems are their low solubility and toxicity. The applicability of CNTs in biological systems [15, $16]$ is critically related to their solubility in aqueous environments. CNTs themselves have a very low solubility in aqueous solutions that had been a major barrier for a variety of potential applications. On the other hand, non-functionalised CNTs are poorly soluble in water [17]. Different studies have shown that CNTs have toxic effects on biological systems [18-20]. Functionalization can decrease the toxicity of CNTs and improves their biocompatibility. Strategic approaches towards increasing the solubility of 
nanotubes have been developed mainly through the surface functionalization.

Functionalization using elemental metal can lead to improved solubility of nanotubes [21]. It has been experimentally shown that metal particles can be adsorbed onto CNTs [22]. Nanotubes can be doped with alkali metals $[23,24]$ and therefore can be considered as guest-host systems. Use of $\mathrm{Li}$ as the mostly used alkali metal in these processes is in situ Raman-studied in detail [25]. The activity of $\mathrm{Li}$ in the reactions with nucleophiles is more than other alkali metal due to the larger amount of $\mathrm{z} / \mathrm{r}$ for Li. Moreover, the weight of Li doped NTs is less than the other alkali metal.

It was shown that $\mathrm{CNT}$ can be doped with $\mathrm{Li}$ to create LiC6 by chemical redox reactions [26, 27]. Quantum mechanical calculation of alkali metal doped carbon nanotubes have been indicated that these structures are stable [28]. The results of monte carlo simulation of metal doped CNTs indicated that alkali metal doping increase the dispersibility of CNTs in aqueous solution [29].

Realizing the fact that metal functionalization can increase the reactivity of the exterior surface of CNTs, we feel that it is highly desirable to study of metal doped CNTs as Possible Carriers in Drug Delivery Systems. Aspirin will be used as a model drug in this research.

Acetylsalicylic acid (ASA), commonly known as Aspirin is a derivative of salicylic acid. Aspirin is a widely used drug. When Aspirin is placed in aqueous solution; it will be hydrolyzed to salicylic acid and acetic acid. Salicylic acid was thought to be too dangerous on the stomach. Components of aspirin are known to soften the cellular membranes by solubilizing the cell's surface proteins. Aspirin is used as an analgesic, antiinflammatory, antipyretic, anticoagulant and antirheumatic and non-steroidal anti-inflammatory drug [30]. It reduces fever by causing the blood vessels in the skin to dilate, allowing heat from the body to leave more rapidly.

We are interested in the interaction of CNTs and aspirin, since the results of loading drugs on to CNTs indicated that the rate of Aspirin released from the prepared CNT-Aspirin composites is slower compared with the commercialized Aspirin [31]. Therefore the CNTs-Aspirin complexe is suitable for slow release of drugs used in cancer therapy and anti platelet agent. A few experimental studies have performed on the interaction of CNT and aspirin and there is no research about metal doped CNTs. The use of CNT as a drug deliver is to musk the drug and makes it inactive, until it reaches the location of action. The transport of drug molecules to the target organ without the loss of its pharmaceutical and activity is the basic purpose of most drug delivery systems. The adsorption of the drug onto CNTs can improve the efficiency of drug transport and also reducing the toxic side effects of the drug.

In this study we investigate the interaction of CNT and Li doped CNT with Aspirin in gas phase and aqueous solution. Quantum mechanical calculations were used for estimating of the interaction energies in gas phase that in the next part followed by the Monte Carlo simulation of the structures in aqueous media. Biocompatibility of Li doped CNTs was then determined. Since CNTs are highly toxic, mainly due to their insolubility, it was verified the solubility of $\mathrm{Li}$ doped CNTs and ASA-Li doped CNTs in physiological media. Perturbation methods were used to calculate solvation free energies and compare the solubility of the complexes. In addition, complexation free energies of CNT-Aspirin complexes were computed to compare the stability of the structures. The validation of our theoretical model is confirmed with experimental results for CNT-drug interactions.

\section{Computational details}

The usage of CNT as a drug delivery system is dependent upon the ability to transport drugs while remaining soluble in a biological system. Therefore, the interactions of Aspirin and CNT in aqueous media were the aim of this research. Then the binding energies and solubility of the structures were studied. The research comprised two sections: quantum mechanics and Monte Carlo simulation.

In the quantum mechanical part, isolated molecules were optimized. The stability of the structures was studied by quantum mechanical calculations. Each species was optimized by the DFT/B3LYP method using the $6-31 \mathrm{G}^{*}[32,33]$ basis set. Vibrational frequency analyses were done to all optimized geometries to confirm that the structures were in the minimum of potential energy surface. The length of the tube has been chosen constant (equal to 0.86 $\mathrm{nm}$ ) which corresponds to five layers of carbon ring hexagons along the tube axis. Then interactions of drug with the central ring were studied. In all 
calculations, nanotubes were capped with hydrogen atoms. All structural optimization were performed by using the GAMESS-US quantum chemistry package [34].

To compare of CNT with metal doped CNT for the use in drug delivery system, the interaction of CNT and also Li doped CNT with Aspirin and Aspirin anion were studied.

Aspirin structure was presented in Fig. 1.

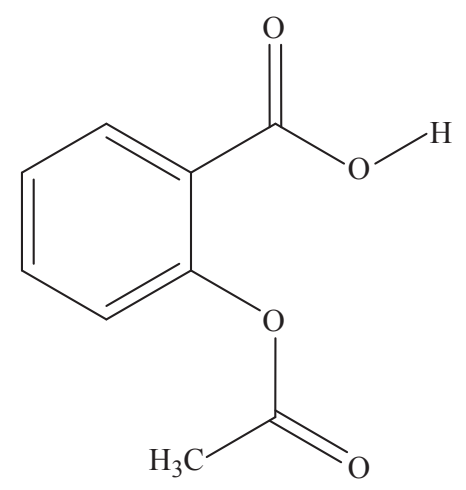

Fig. 1 Aspirin structure.

Four possible interactions were studied: interactions of CNT and Li doped CNT with $\mathrm{COOH}$ group of Aspirin and interactions of CNT and $\mathrm{Li}$ doped CNT with $\mathrm{COO}^{-}$group of Aspirin anion. The optimized structures of Aspirin complexes were indicated in Fig. 2.

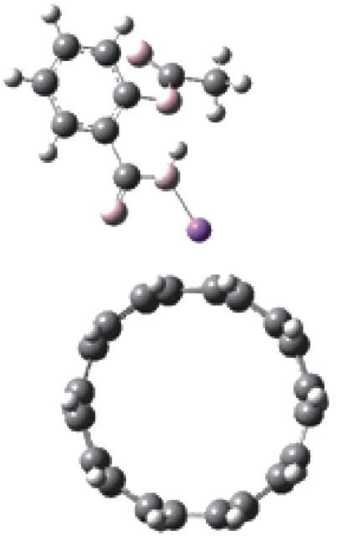

(a)

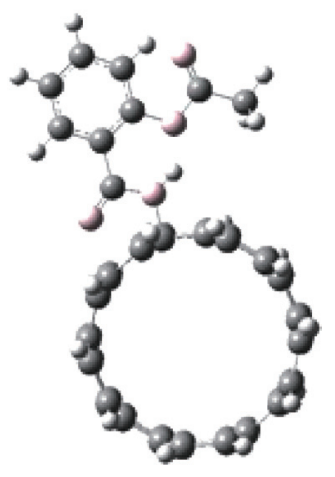

(b)
Fig. 2 Structure of (a) CNTLi-Aspirin (b) CNT-Aspirin.

In the subsequent simulation step, these geometries and quantum mechanical charges were applied.

It was applied Monte Carlo simulation for dilute solutions of nanotubes and drug in water. The monomers were represented by interaction sites usually located on nuclei. The interaction energy between two molecules was expressed by pair wise sum of interaction contributions. The Transferable Intermolecular Potential function (TIP3) [35, 36] was applied for modeling water molecules, and standard Lennard-Jones (LJ) potential to present the short range potential and a long range Columbic potential, with parameters $\varepsilon, \sigma$ and $\mathrm{q}$ for each atoms in nanotubes. For both models, the pair potential function $\mathrm{E}_{\mathrm{ij}}$ was represented by Coulombic and Lennard-Jones terms between sites centered on nuclei:

$$
E^{A B}=\sum_{i}^{o n A} \sum_{j}^{o n B} 4 \varepsilon_{i j}\left[\left(\frac{\sigma_{i j}}{r_{i j}}\right)^{12}-\left(\frac{\sigma_{i j}}{r_{i j}}\right)^{6}\right]+\frac{q_{i} q_{j} e^{2}}{r_{i j}}
$$

The Lennard-Jones parameters between pairs of different atoms are obtained from the LorentzBerthelodt combination rules. Appropriate LennardJones parameters for Carbon [37], Li [38] and atom types in Aspirin [39, 40] have been used in the calculations. These parameters are given in Table 1.

Table 1 Lennard-Jones Parameters for the atoms in CNT and drug

\begin{tabular}{ccc}
\hline Site & $\varepsilon, \mathrm{kcal} / \mathrm{mol}$ & $\sigma, \AA$ \\
\hline $\mathrm{C}$ & 0.08 & 3.5 \\
$\mathrm{O}$ & 0.21 & 2.96 \\
$\mathrm{C}$ in C=O & 0.105 & 3.75 \\
other C & 0.08 & 3.5 \\
$\mathrm{H}$ on C & 0.05 & 2.5 \\
$\mathrm{Li}$ & 0.0183 & 2.13 \\
\hline
\end{tabular}

In all separate $\mathrm{MC}$ simulations performed here, a standard manner the Metropolis sampling technique [41] in canonical (T, V, N) ensemble was used. All calculations were performed in a cubic box at the experimental density of water, $0.993 \mathrm{~g} / \mathrm{cm}^{3}$ at $37^{\circ} \mathrm{C}$ [42]. The optimum edges of the box were $5 \mathrm{~nm}$, which corresponds to almost $4000 \mathrm{H}_{2} \mathrm{O}$ molecules of pure solvent. The symmetry center of the nanotube was in the geometrical center of the cell. An acceptance rate of $50 \%$ for new configurations was achieved by using suitable ranges for translations and rotation about a randomly chosen axis. Periodic boundary conditions were employed in computation. The system was thoroughly equilibrated using several hundred thousand configurations.

To calculate the solvation free energies of molecules, the thermodynamic perturbation method was applied in these computations. Appropriate steps were taken to obtain the most accurate results possible 
with a molecular mechanical based approach. The Zwanzig equation [43] was used to evaluate free energy changes:

$\Delta G(A \rightarrow B)=G_{B}-G_{A}=-R T \ln \left\langle\exp -\left(E_{B}-E_{A}\right) / R T\right\rangle$

In this equation, $\mathrm{A}$ and $\mathrm{B}$ indicate the reference and the perturbed states, and the symbol \langle\rangle$_{A}$ indicates that sampling has been carried out in the reference state.

The solvation free energy $\left(\Delta G_{\text {sol }}(\mathrm{A})\right)$ is defined as the free energy of transfer of one mole of solute (A) from an ideal gas state to an infinitely dilute solution. This free energy of transfer is the difference between the free energies of $\mathrm{A}$ in the two phases.

\section{Results and discussions}

In current research, we studied the interaction of Aspirin with CNT and Li doped CNT. Carboxyl group of aspirin and aspirin anion were considered to interact with CNT and Li doped CNT. The binding energies $\left(E_{\mathrm{b}}\right)$ are defined as the energy difference between the energy summation of two individually optimized monomers and the fully optimized complexes. The $E_{\mathrm{b}}$ is defined as following:

$E_{\mathrm{b}}=E_{\mathrm{CNT}-\mathrm{D}}-\left(E_{\mathrm{CNT}}+E_{\mathrm{D}}\right)+\mathrm{BSSE}$

where $E_{\mathrm{D}}$ is the energy of an isolated drug (Aspirin or Aspirin anion), $E_{\mathrm{CNT}}$ is the total energy of $\mathrm{CNT}$ (or Li doped CNT) in the absence of the adsorbed species, $E_{\mathrm{CNT}-\mathrm{D}}$ is the energy of Aspirin and CNT complex, and BSSE is basis set superposition error correction [44]. By definition, $E_{\mathrm{b}}<0$ in Eq. (3) corresponds to a stable complex of drug and CNT.

Their energetic properties are shown in Table 2. As can be seen, the binding energy of the interaction of ASA and $\mathrm{ASA}^{-}$with CNT is $12.18 \mathrm{eV}$ and $8.79 \mathrm{eV}$

Table 2 Quantum mechanical results in gas phase

\begin{tabular}{cccc}
\hline Species & Stoichiometry & Energy $(\mathrm{eV})$ & $\begin{array}{c}\text { Binding } \\
\text { energy }(\mathrm{eV})\end{array}$ \\
\hline $\mathrm{CNT}$ & $\mathrm{C}_{80} \mathrm{H}_{20}$ & -83266.76 & - \\
$\mathrm{CNTLi}$ & $\mathrm{C}_{80} \mathrm{H}_{20} \mathrm{Li}$ & -83471.37 & - \\
$\mathrm{ASA}$ & $\mathrm{C}_{9} \mathrm{H}_{8} \mathrm{O}_{4}$ & -17645.69 & - \\
ASA $^{-}$ & $\mathrm{C}_{9} \mathrm{H}_{7} \mathrm{O}_{4}$ & -17629.94 & - \\
CNTASA & $\mathrm{C}_{89} \mathrm{O}_{4} \mathrm{H}_{28}$ & -100900.27 & 12.18 \\
CNT-ASA $^{-}$ & $\mathrm{C}_{89} \mathrm{O}_{4} \mathrm{H}_{27}$ & -100887.91 & 8.79 \\
CNTLi-ASA $^{-}$ & $\mathrm{C}_{89} \mathrm{O}_{4} \mathrm{H}_{28} \mathrm{Li}$ & -101117.76 & -0.69 \\
CNTLi-ASA $^{-}$ & $\mathrm{C}_{89} \mathrm{O}_{4} \mathrm{H}_{27} \mathrm{Li}$ & -101104.14 & -2.82 \\
\hline
\end{tabular}

respectively. The binding energy of the interaction of ASA and $\mathrm{ASA}^{-}$with Li doped CNT is $-0.69 \mathrm{eV}$ and $-2.82 \mathrm{eV}$ respectively. So it is predicted that the complexes of Aspirin and aspirin anion with CNT are not stable. In fact aspirin could not interact with non functionalized CNTs. The achievement of drug-CNT interaction is dependent upon several factors factors such as the reactivity of the CNT, the reactivity of the drug, etc. [45]. Metal doping increases the reactivity of CNT that cause the enhancement of binding energy with drug. Therefore, Li doped CNT can be adsorb Aspirin to act as a carrier for it.

Our results indicate that Aspirin anion adsorbs onto Li doped CNT with $2.13 \mathrm{eV}$ binding energy more than neutral Aspirin. The reactivity of $\mathrm{ASA}^{-}$is more than ASA for the interaction with Li doped CNT. So ASA ${ }^{-}$ makes more stable complex with CNTLi.

These results were obtained in gas phase. But we interested in the interaction of CNT with drug in physiological solution. Therefore in the next stage, these interactions were studied in aqueous solution. For this purpose, the solvation of Aspirin, CNT and their complexes were studied in the presence of water. Then free energies of complex formation were obtained to study the possibility of the interactions in aqueous solution.

The process of solvation of one molecule in water can be defined as transferring it from gas phase to aqueous solution. One molecule of solute was merged in water and average energies were calculated from Monte Carlo simulations.

Total energies which were calculated by Monte Carlo simulations are given in Table 3. This table also includes the number of solvent molecules $\left(\mathrm{N}_{\mathrm{H}_{2} \mathrm{O}}\right)$ in the cubic box, the energy contributions from solute solvent interaction energy $\left(E_{\text {soln }}\right)$ and Electrostatic and van der Waals contributions in $\mathrm{E}_{\text {soln }}$.

The results indicated that the absolute total energies of CNT were less than complexes of CNT and Aspirin. The absolute energy of CNT-ASA ${ }^{-}$in water is larger than CNT-ASA and absolute energy of CNTLi-ASA ${ }^{-}$ in water is larger than CNTLi-ASA. Moreover, the absolute total energy of the complexes of CNTLi and Aspirin in water is larger than CNT and Aspirin.

The presence of electrostatic charges and adsorption of various molecules on the surface brings in large changes in the properties of the CNT structure. Intermolecular interactions (including 
Table 3 Simulation results in water media

\begin{tabular}{|c|c|c|c|c|c|c|}
\hline species & $\mathrm{N}_{\mathrm{H}_{2} \mathrm{O}}$ & $\langle\mathrm{E}\rangle\left(\mathrm{kcal} \mathrm{mol}^{-1}\right)$ & $\mathrm{E}_{\text {soln }}$ & $\begin{array}{c}\text { Electrostatic } \\
\text { contribution in } \mathrm{E}_{\text {soln }}\end{array}$ & $\begin{array}{l}\text { van der Waals } \\
\text { contribution in } \mathrm{E}_{\text {soln }}\end{array}$ & $\begin{array}{l}\text { Drug contribution in } \\
\qquad \mathrm{E}_{\text {soln }}\end{array}$ \\
\hline $\mathrm{CNT}$ & 4047 & -9.451 & -0.302 & 0 & -0.302 & - \\
\hline CNTLi & 4045 & -17.967 & -14.473 & -14.375 & -0.099 & - \\
\hline ASA & 4068 & -9.487 & -0.551 & -0.526 & -0.026 & - \\
\hline $\mathrm{ASA}^{-}$ & 4070 & -10.529 & -2.227 & -2.229 & 0.002 & - \\
\hline CNT-ASA & 4036 & -13.398 & -7.128 & -6.914 & -0.215 & -0.376 \\
\hline CNT-ASA $^{-}$ & 4036 & -14.665 & -9.264 & -9.050 & -0.213 & -0.713 \\
\hline CNTLi-ASA & 4031 & -13.927 & -7.722 & -7.506 & -0.216 & -0.146 \\
\hline CNTLi-ASA $^{-}$ & 4031 & -15.317 & -10.061 & -9.846 & -0.215 & -1.668 \\
\hline
\end{tabular}

van der Waals and electrostatic interactions) have important contribution in total energy and the atomic charges affect on the electrostatic term of total energy. Quantum mechanical calculations indicated that the atoms in CNT-Aspirin complexes carry partial charges due to the interaction of Aspirin with CNT. For better evaluation, electrostatic and van der Waals contributions of potential energy of the systems were also computed. As can be seen in Table 3, in CNTAspirin complexes, electrostatic contribution of the solute-water interaction energy is larger than van der Waals contribution due to the partial atomic charges in the complexes. Pure CNT have no atomic charge and van der Waals term has dominate contribution in solvation. Therefore $E_{\text {soln }}$ and total energy of the complexes must be larger than Pure CNT. All of the Aspirin complexes have approximately the same van der Waals contribution in total energy. Therefore the differences of total energy in solvent are related to the electrostatic contribution due to the atomic charges. Among CNT-Aspirin complexes, electrostatic contribution of the solute-water interaction energy for $\mathrm{CNT}_{-\mathrm{ASA}^{-}}\left(-9.05 \mathrm{kcal} \mathrm{mol}^{-1}\right)$ is greater than CNTASA (-6.914 kcal mol $\left.{ }^{-1}\right)$. Between CNTLi-Aspirin complexes, electrostatic contribution of the solutewater interaction energy for CNTLi-ASA ${ }^{-}(-9.846 \mathrm{kcal}$ $\left.\mathrm{mol}^{-1}\right)$ is greater than CNTLi-ASA $\left(-7.506 \mathrm{kcal} \mathrm{mol}^{-1}\right)$. Between CNT and CNTLi complexes, total energy of CNTLi complexes in solution is larger than CNT complexes due to the greater electrostatic interactions between CNTLi-Aspirin complexes and water. Of course the total energy of CNTLi in water is larger than CNTLi-Aspirin complexes. It is attributed to the charge distribution of Li and Aspirin in complexes.
To study of the drug effect in the intermolecular interaction of CNT and solvent, the drug contribution in the solute-solvent interaction energy was also reported in Table 3. It reveals that drug (Aspirin) contributions on CNT-water interaction energy for the solutes, CNT-ASA and CNT-ASA ${ }^{-}$are -0.376 and $-0.713 \mathrm{kcal} \mathrm{mol}^{-1}$, and also for CNTLi-ASA and CNTLi-ASA ${ }^{-}$, are $-0.146,-1.668 \mathrm{kcal} \mathrm{mol}^{-1}$ respectively. These results indicated that Aspirin anion is more effective form of Aspirin for increasing the intermolecular interaction with water. So it is predicted

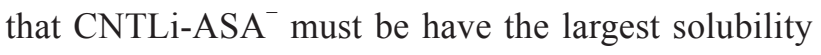
in water.

To confirm these consequences and to evaluate the solubility of the CNTs, we need to estimate the solvation free energies of the structures. Therefore solvation free energies of the CNTs were calculated in the next step.

Solvation free energy calculations for each of the solutes (CNT, CNT-Aspirin complexes and CNTLiAspirin complexes) were carried out. To model the solvation of CNT and its complexes, a simulation was performed on the system with the solute fully represented and then it's electrostatic and van der Waals parameters decreased to zero. The computed solvation free energies, $\Delta G_{\text {sol }}$, are presented in Table 4. It could be seen in Table 4; CNT had the smaller solvation free energy than complexes. Computed solvation free energies indicated that complex formation with Aspirin increase the solvation of CNT in water. The solvation of CNT increases significantly by the interaction with Aspirin. In fact, after interaction of drug with CNT, solvation free energy increases from 
Table 4 Solvation free energies $\left(\Delta G_{\text {sol }}\right)$ and complexation free energies $\left(\Delta G_{\mathrm{r}}\right)$ of aspirin-CNT complexes

\begin{tabular}{ccc}
\hline species & $\Delta G_{\text {sol }}\left(\mathrm{kcal} \mathrm{mol}^{-1}\right)$ & $\Delta G_{\mathrm{r}}\left(\mathrm{kcal} \mathrm{mol}^{-1}\right)$ \\
\hline CNT & -2.97 & - \\
CNTLi & -99.967 & - \\
ASA & -4.259 & - \\
ASA $^{-}$ & -15.508 & - \\
CNTASA & -48.648 & 41.419 \\
CNTASA $^{-}$ & -63.355 & 44.877 \\
CNTLiASA $^{\text {CNTLiASA }}$ & -53.395 & -50.831 \\
\hline
\end{tabular}

-2.970 up to -48.648 and -63.355 for CNT-ASA and $\mathrm{CNT}_{-\mathrm{ASA}^{-}}^{-}$respectively. So it is predicted that drug has the large contribution in increasing of the solvation of CNT. This is related to the larger electrostatic contribution and also larger intermolecular interactions between solute-solvent molecules.

For the CNTLi, interactions with Aspirin decrease the solvation free energies. According to the calculated electrostatic contribution of total energy (showed in Table 3), CNTLiASA and CNTLiASA ${ }^{-}$have smaller electrostatic interactions with solvent. Therefore solvation of CNTLi is naturally greater than its complexes. Besides, we considered three Aspirin species: Aspirin, CNT-Aspirin and CNTLi-Aspirin. Solvation free energy of CNTLi-Aspirin is larger than CNT-Aspirin and Aspirin itself. Consequently, among these possible Aspirin structures, CNTLi-Aspirin is the most effective form for producing the best soluble drug-CNT species in aqueous solution.

We also computed complexation free energies $\left(\Delta G_{\mathrm{r}}\right)$ in aqueous solutions. For this purpose, we need these solvation free energies: solvation free energy of Aspirin species, CNT, CNTLi, CNT-Aspirin complexes and CNTLi-Aspirin complexes. The results are shown in Table 4. It can be seen that the complexation free energies of CNT-Aspirin complexes are positive. So it is concluded that nonfunctionalized CNT cannot interact with Aspirin in Aqueous media. This conclusion is compatible with experimental result of the interaction of multi-walled CNT with Aspirin [27]. Drugs have been only adsorbed onto the functionalized CNT. Yusof et al. have modified Surface structure of CNT using basic functionalization techniques namely oxidation with concentrated acid to generate functionalized multi-walled CNT. Then loading of drug onto the functionalized CNT could be performed. The quantitative determination of aspirin adsorbed onto the functionalized CNT has been done by fluorescence spectrometry. The results indicated that the aspirin molecules may be bonded with the carboxylic functional groups on the surface of nanotubes.

Our study indicated that only CNTLi can interact with ASA and $\mathrm{ASA}^{-}$with the complexation free energies of -50.831 and $-45.809 \mathrm{kcal} \mathrm{mol}^{-1}$ respectively. Therefore CNTLi is a suitable compound to transport Aspirin in aqueous media. This consequence seems to be compatible with the gas phase result.

\section{Conclusions}

Due to the potential applications of CNTs in drug delivery and medical science, the study of the interaction of Aspirin with CNT and CNTLi in gas phase and aqueous solution was the aim of this research.

Two parts of this research were focused on quantum mechanical calculation and Monte Carlo simulation. In quantum mechanical part, binding energies of Aspirin species with CNT and CNTLi were calculated in gas phase. Two possible form of Aspirin were considered: Aspirin anion and neutral Aspirin. The calculations showed that only CNTLi can bind to Aspirin effectively and, Aspirin anion has the most interaction energy.

In Monte Carlo section, solvation free energies of the complexes were estimated by free energy perturbation method. The computations showed that the solvation of CNTLi-Aspirin complexes in water was larger than CNT-Aspirin.

Computed complexation free energies of these complexes in physiological media indicated that CNTLi-Aspirin complexes had negative complexation free energy, therefore it is stable compound in water .

Consequently, CNTLi can effectively interact with Aspirin species. CNTLi display low toxicity due to the better solubility in aqueous media. In fact CNTLi can be adsorbed Aspirin as a drug, and can be used to deliver it to cells.

\section{References}

[1] C.V. Nguyen, L. Delzeit, A.M. Cassell, et al., Meyyappan. 
Preparation of nucleic acid functionalized carbon nanotube arrays. Nano Lett., 2002, 2: 1079-1081.

[2] Y. Lin, S. Taylor, H.P. Li, et al., Advances toward bioapplicationsofcarbon nanotubes. J. Mater. Chem., 2004, 14: 527-541.

[3] A. Bianco, K. Kostarelos, M. Prato, Applications of carbonnanotubes in drug delivery. Curr. Opin. Chem. Biol., 2005, 9: 674-679.

[4] N.W.S. Kam, H.J. Dai. Carbon Nanotubes as intracellular proteintransporters: generality and biological functionality. J. Am. Chem. Soc., 2005, 127: 6021-6026.

[5] S. Ketabi, S.M. Hashemianzadeh, M.M. Waskasi. Study of DNA base- Li doped SiC Nanotubes in aqueous solutions: A Computer Simulation Study. J. Mol. Model. ,2013, 19: 1605-1615.

[6] N.W.S. Kam, M. O'Connell, J.A. Wisdom, H.J. Dai, Carbon nanotubes as multifunctional biological transporters and nearinfrared agents for selective cancer cell destruction. Proc Natl Acad Sci USA, 2005, 102 $11600-11605$

[7] N.W.S. Kam, Z.A. Liu, H.J. Dai. Carbon nanotubes asintracellular transporters for proteins and DNA: an investigation of theuptake mechanism and pathway. Angew. Chem. Int Ed., 2006, 45: 577-581.

[8] A.A. Bhirde, V. Patel, J. Gavard, et al., Targeted killing of cancer cells in vivo and in vitro with EGF- directed carbon nanotube- based drug delivery. ACS Nano., 2009, 3: $307-316$.

[9] D. Chen, X. Wu, J. Wang, et al., Morphological Observation of Interaction between PAMAM Dendrimer Modified Single Walled Carbon Nanotubes and Pancreatic Cancer Cells. Nano Biomed. Eng., 2010, 2: 61-66.

[10] D. Cui, H. Zhang, J. Sheng, et al., Effects of CdSe/ZnS quantum dots covered multi-walled carbon nanotubes on murine embryonicstem cells. Nano Biomed. Eng., 2010, 2 236-244.

[11] Z. Tian, Y. Shi, M. Yin, et al., Functionalized Multiwalled Carbon Nanotubes-anticancer Drug Carriers: Synthesis, Targeting Ability and Antitumor activity. Nano Biomed Eng., 2011, 3: 157-162.

[12] V.S. Thakare, M. Das, A.K. Jain, et al., Carbon nanotubes in cancer theragnosis. Nanomedicine, 2010, 5: $1277-$ 1301.

[13] R.J. Chen, S. Bangsaruntip, K.A. Drouvalakis, et al., Noncovalent Functionalization of Carbon Nanotubes for Highly Specific Electronic Biosensors. Proc. Natl. Acad. Sci., U.S.A., 2003, 100: 4984-4989.

[14] W.F. DeGrado, G. Grigoryan, Y.H. Kim, et al., Computational design of virus-like protein assemblies on carbon nanotube surfaces. Science, 2011, 332: 10711076.

[15] Y. Lin, S. Taylor, H. Li, et al., Advances toward bioapplications of carbon nanotubes. J. Mater Chem., 2004, 14: 527-541.

[16] A. Das, A.K. Sood, P.K. Maiti, et al., Binding of nucleobases with single-walled carbon nanotubes: theory and experiment. Chem Phys Lett., 2008, 453: 266-273.

[17] H.H. Haeri, S. Ketabi, S.M. Hashemianzadeh. The solvation study of carbon, silicon and their mixed nanotubes in water solution. J. Mol. Model., 2012, 18: 3379-3388.

[18] H.J. Johnston, G.R. Hutchison, F.M. Christensen, et al., A critical review of the biological mechanisms underlying the in vivo and in vitro toxicity of carbon nanotubes: the contribution of physico-chemical characteristics. Nanotoxicology, 2010, 4: 207-246.

[19] C.W. Lam, J.T. James, R. McCluskey, et al., A review of carbon nanotube toxicity and assessment of potential occupational and environmental health risks. Crit Rev Toxicol, 2006, 36: 189-217.
[20] L.E. Murr, K.M. Garza, K.F. Soto, et al., Cytotoxicity assessment of some carbon nanotubes and related carbon nanoparticle aggregates and the implications for anthropogenic carbon nanotube aggregates in the environment. Int. J. Environ. Res. Public Health, 2005, 2: $31-42$.

[21] K.B.I. Kharisov, O.V. Kharissova, H.L. Gutierrez, et al., Recent advances on the soluble carbon nanotubes. Ind. Eng. Chem. Res., 2009, 48: 572-590.

[22] G.G. Wildgoose, C.E. Banks, R.G. Compton, Metal nanoparticles and related materials supported on carbon nanotubes: methods and application. Small, 2006, 2: 182-193.

[23] O. Zhou, R.M. Fleming, D.W. Murphy, et al., Defects in carbon nano-structures. Science, 1994, 263: 1744-1747.

[24] J.E. Fischer, R.S. Lee, H.J. Kim, et al., Conductivity enhancement in single walled carbon nanotube bundles doped with K and Br. Nature, 1997, 388: 255-257.

[25] Z. Gu, F. Liang, Z. Chen, et al., In situ Raman studies on lithiated single-wall carbon nanotubes in liquid ammonia. Chem. Phys. Lett., 2005, 410, 467-470.

[26] E. Jouguelet, C. Mathis, P. Petit, Controlling the electronic properties of single-wall carbon nanotubes by chemical doping. Chem. Phys. Lett., 2000, 318: 561-564.

[27] A.S. Claye, J.E. Fischer, C.B. Huffman, et al., Solid-state electrochemistry of the Li single wall carbon nanotube system. J. Electrochem Soc., 2000, 147: 2845-2852.

[28] S. Zeynali, S. Ketabi, H.R. Aghabozorg. Density Functional Study of Hydrogen Adsorption on Alkali Metal Doped Carbon Nanotube. J. Comput. Theor. Nanosci., 2014, 11: 1-6.

[29] S. Ketabi, M. Aarabmaghsoudi. Effect of metal doping on the solvation of carbon nanotubes: a Monte Carlo simulation study. Physics and Chemistry of Liquids, 2014, 52: 763-776

[30] F. Catella-Lawson, M.P. Reilly, S.C. Kapoor, et al., Cyclooxygenase inhibitors and the antiplatelet effects of aspirin. New England Journal of Medicine, 2001, 345: 1809-1817.

[31] A.M. Yusof, N.A. Buang, L.S. Yean, M.L. Ibrahim, et al., The use of multi-walled carbon nanotubes as possible carrier in drug delivery system for aspirin. AIP Conf. Proc., 2009, 1136: 390-394.

[32] G.A. Petersson, M.A. Al-Laham. A complete basis set model chemistry. II. Open-shell systems and the total energies of the first-row atoms. J. Chem. Phys., 1991, 94: 6081-6090.

[33] G.A. Petersson, A. Bennett, T.G. Tensfeldt, et al., Complete basis set model chemistry. I. The total energies of closed-shell atoms and hydrides of the first-row elements. J. Chem. Phys., 1988, 89: 2193-2218.

[34] M.W. Schmidt, K.K .Baldridge, J.A. Boatz, et al., General Atomic and Molecular Electronic Structure System. $J$. Comput. Chem., 1993, 14: 1347-1363.

[35] W.L. Jorgensen, J. Chandrasekhar, J.D. Madura, et al., Comparison of simple potential functions for simulating liquid water. J. Chem. Phys., 1983, 79: 926-935.

[36] W.L. Jorgensen. Transferable intermolecular potential functions for water, alcohols and ethers, Application to liquid water. J. Am. Chem. Soc., 1981, 103: 335-340.

[37] Y. Jia, M. Wang, L. Wu, C. Gao. Separation of $\mathrm{CO}_{2} / \mathrm{N}_{2}$ Gas Mixture through Carbon Membranes: Monte Carlo Simulation. Separation Science and Technology, 2007, 42: 3681-3695.

[38] J. Chandrasekhar, D.C. Spellmeyer, W.L. Jorgensen, Energy component analysis for dilute aqueous solutions of lithium(1+), sodium(1+), fluoride(1-), and chloride(1-) ions. J. Am. Chem. Soc., 1984: 903-910.

[39] W.L. Jorgensen, C.J. Swenson. Optimized intermolecular potential functions for amides and peptides. Hydration of 
amides. J Am Chem Soc., 1985, 107: 1489-1496.

[40] R.C. Rizzo, W.L. Jorgensen, OPLS All-Atom Model for Amines: Resolution of the Amine Hydration Problem. $J$. Am. Chem. Soc., 1999, 121: 4827-4836.

[41] N. Metropolis, A.W. Rosenbluth, M.N. Rosenbluth, et al., Equation of state calculations by fast computing machines. J. Chem. Phys., 1953, 21: 1087-1093.

[42] K.N. Marsh, Recommended Reference Materials for the Realization of Physicochemical Properties. Blackwell Scientific Publications, Oxford, 1987.

[43] R.W. Zwanzig. High temperature equationof state by perturbation method. J. Chem. Phys., 1954, 22: 14201422.

[44] S.F. Boys, F. Bernardi, The calculation of small molecular interactions by the differences of separate total energies. Some procedures with reduced errors. Mol. Phys., 1970, 19: 553-566.

[45] M. Menon, E. Richter, A. Mavrandonakis, et al., Structure and stability of SiC nanotubes. Phys. Rev. B, 2004, 69: 115322-115334.

Copyright $₫ 2015$ Yasaman Ketabi and Sepideh Ketabi. This is an open-access article distributed under the terms of the Creative Commons Attribution License, which permits unrestricted use, distribution, and reproduction in any medium, provided the original author and source are credited. 